\title{
ORTODONCIA Y ERUPCIÓN PASIVA
}

\author{
Juan Esteban Rendón Giraldo*, Odon. ${ }_{1}$, Andrea Pedroza Garcés, Odon. ${ }_{2}$ \\ ${ }_{1}$ Universidad de Antioquia, Universidad Cooperativa de Colombia, sede Medellín, Colombia \\ ${ }_{2}$ Universidad CES, Universidad Cooperativa de Colombia, sede Medellin, Colombia
}

Recibido: 2 de noviembre del 2012 Aprobado: 21 de febrero del 2013

* Autor de contacto: Juan Esteban Rendón, Facultad de Odontología, Universidad Cooperativa de Colombia, 2706466, Carrera 47 \# 37 sur 18, Medellín, Colombia correo electrónico: juanesregi@hotmail.com

Cómo citar este artículo: Rendón Giraldo JE, Pedroza Garces A. Ortodoncia y erupción pasiva. Rev. Nac. Odontol. 2013 diciembre; 9 (edición especial): $101-108$.

Resumen. La erupción pasiva alterada (EPA) es una condición periodontal que puede alterar significativamente el logro de los objetivos estéticos del tratamiento de ortodoncia. Aunque esta alteración es conocida por los ortodoncistas, la mayoría de ellos no reconocen la diferencia en cuanto a su clasificación y manejo. Por tanto, la intención de este artículo es describir los lineamientos para realizar un diagnóstico certero, explicar su tratamiento y establecer un protocolo de manejo para la EPA, que indique en qué momento remitir para que se realicen los procedimientos plásticos periodontales, basado en los hallazgos clínicos y teniendo en cuenta la edad como factor crítico para definir el procedimiento.

Palabras clave: edad, erupción pasiva alterada, estética gingival, ortodoncia.

\section{Ortodontia and Passive ERUption}

Abstract. Altered Passive Eruption (APE) is a condition that can significantly affect achievement of the aesthetic goals of orthodontic treatment. Although this condition is known to orthodontists, most do not recognize the difference in its classification and management. The aim of this article is therefore to describe the guidelines for accurate diagnosis, explain the treatment and establish a management protocol for ape that would indicate when such patients are to be referred for plastic periodontal procedures, based on clinical findings and considering age as a critical factor when determining the procedure.

Keywords: age, altered passive eruption, gingival aesthetics, orthodontia. 


\section{Introducción}

La erupción dentaria está integrada por una fase activa y una pasiva. La erupción activa es el movimiento de los dientes en dirección al plano oclusal [1], mientras que la erupción pasiva es la exposición de los dientes por la migración apical de la encía [2, 3], aceptándose que el margen gingival en un adulto que no haya tenido pérdida de inserción se posiciona a nivel, o a 1 mm coronal al límite amelo-cementario (LAC) [4]. Así pues, según se produce el proceso de erupción pasiva, aumenta la longitud de la corona clínica [5].

En algunos pacientes los tejidos no logran migrar a una situación próxima al LAC, quedando el margen gingival ubicado incisal u oclusalmente sobre la corona anatómica, lo que resulta en coronas clínicas cortas debido al exceso gingival que cubre la corona anatómica. Esta condición es conocida como erupción pasiva alterada (EPA) [7-9], y aunque su etiología no es clara, la existencia de un periodonto grueso se encuentra asociada con esta condición [10].

La erupción pasiva alterada tiene implicaciones sobre la estética, sobre el periodonto y sobre el logro de resultados ortodóncicos adecuados, así:

- Implicaciones estéticas

- Altera la proporción ancho-largo de la corona: al estar el tejido posicionado coronalmente, hace que la forma coronal se vea cuadrada y sin un adecuado contorno gingival $[11,12]$.

- Sonrisa gingival: el exceso de tejido se tiende a exhibir debajo del borde inferior del labio superior, lo cual hace que muchos pacientes se sientan incómodos al sonreír [11].

- Implicaciones periodontales

- Laceraciones de la encía: el margen gingival, por estar ubicado en una porción inferior y más convexa de la corona, tiene una protección disminuida al traumatismo de la función masticatoria $[9,13,14]$.

- Enfermedad periodontal: aunque la encía de los pacientes con erupción pasiva alterada generalmente es saludable en ausencia de placa [15], el exceso gingival puede dar lugar a la formación de seudobolsas (bolsas gingivales) que, en combinación con una mala higiene oral, pueden ser invadidas por bacterias y así dar inicio a procesos inflamatorios $[7,13,16]$.

- Implicaciones ortodóncicas
- El margen gingival, por estar posicionado coronalmente y no contar con un adecuado festoneado, puede afectar el logro de los resultados ortodóncicos por la imposibilidad de:

a. Ubicar adecuadamente el cenit gingival.

b. Posicionar simétricamente los márgenes gingivales.

El manejo de la erupción pasiva alterada en el paciente ortodóncico requiere un enfoque interdisciplinario entre el ortodoncista y el periodoncista $[7,8,17]$, en el que ambos tengan la capacidad de reconocerla, saber las implicaciones que tiene en el logro de los resultados ortodóncicos adecuados, y determinar el momento apropiado en que esta entidad debe ser manejada.

Con este artículo se pretende que el ortodoncista esté en la capacidad tanto de identificar los signos clínicos de la erupción pasiva alterada, como de conocer la forma en que se debe diagnosticar, clasificar y manejar en el paciente ortodóncico.

\section{Signos clínicos}

La literatura describe signos gingivales, coronales y condiciones de edad que están relacionados con la presencia de erupción pasiva alterada.

\section{Signos gingivales}

\section{Forma y posición gingival}

La forma gingival se refiere al festoneado del margen gingival que es determinado por la relación entre el límite amelo cementario y la cresta ósea. De acuerdo con la Academia Americana de Odontología Cosmética, se acepta que la forma gingival de los incisivos mandibulares y de los laterales superiores debe ser ovalada y simétrica, mientras que la de los centrales y caninos superiores debe ser elíptica y con el cenit ubicado ligeramente hacia distal. Igualmente los márgenes gingivales de los centrales y caninos deben estar a la misma altura, y ligeramente superior que el margen de los laterales [18, 19] (figura 1). Como podemos ver, las características de forma y posición gingival son bastante específicas y tienen gran impacto en la estética dentogingival, pero en los pacientes que tienen erupción pasiva alterada la encía tiene el festoneado prácticamente perdido conservando la misma altura en todo el segmento anterior (figura 2). 


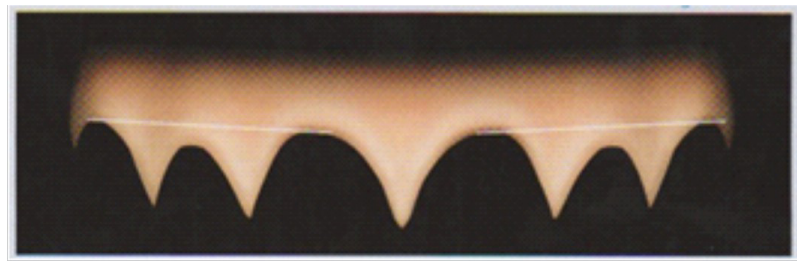

Figura 1. Características ideales de forma y posición de márgenes gingivales

Fuente: Fradeani y Barducci, 2011 [20]

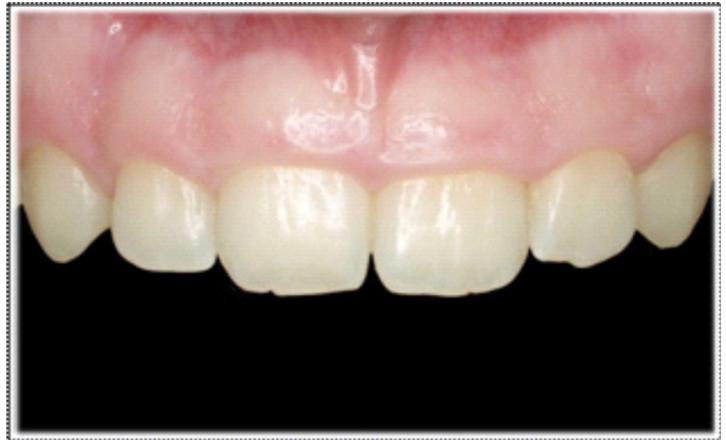

Figura 2. Márgenes en erupción pasiva alterada Fuente: Casos clínicos/periodoncia [21]

\section{Altura de la papila}

La altura de la papila se define como la distancia que existe desde la punta de la papila hasta la parte más profunda del margen gingival $[22,23]$ y se acepta que, en normalidad, su tamaño a nivel de los centrales superiores debe estar entre $4,5 \mathrm{~mm}$ a $5 \mathrm{~mm}$, lo cual coincide con la mitad de la longitud de la corona clínica (figura 3). Generalmente en la erupción pasiva alterada la papila presenta una altura disminuida, lo cual se atribuye al posicionamiento coronal del margen gingival (figura 4).

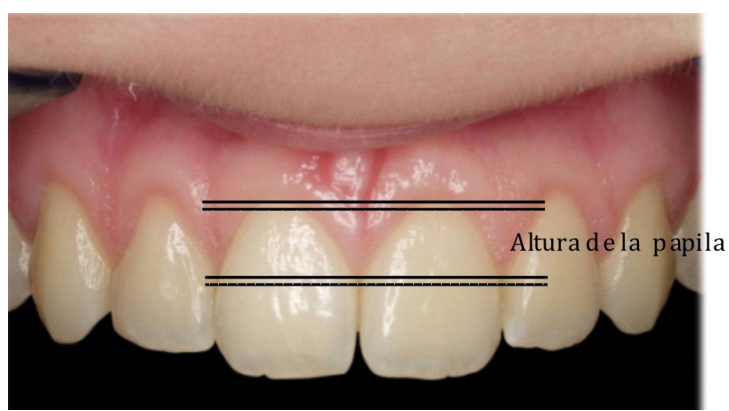

Figura 3. Altura de la papila en paciente sin alteración de erupción Fuente: Casos clínicos/periodoncia [21]

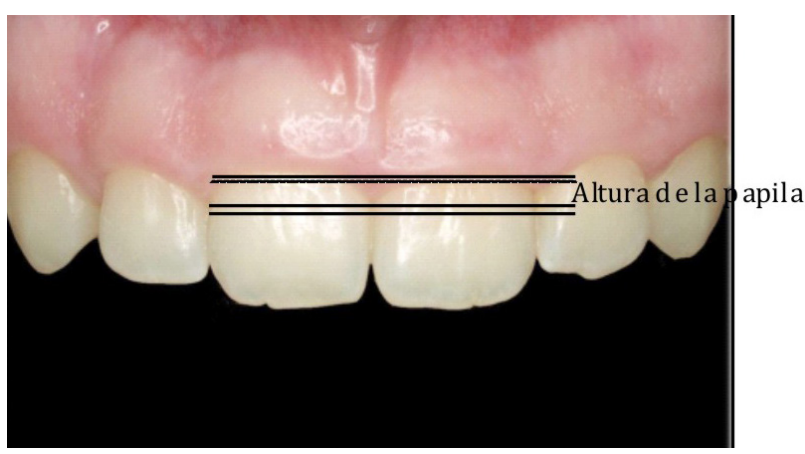

Figura 4. Altura de la papila en paciente con erupción pasiva alterada

Fuente: Casos clínicos/periodoncia [21]

\section{Laceraciones gingivales}

Un margen gingival con enrojecimiento y pequeños desgarros debe alertar al clínico de la posible presencia de una EPA, ya que al estar el margen gingival ubicado en una porción inferior y más convexa de la corona, tiene una protección disminuida al traumatismo de la función masticatoria $[9,13,14]$ (figura 5).

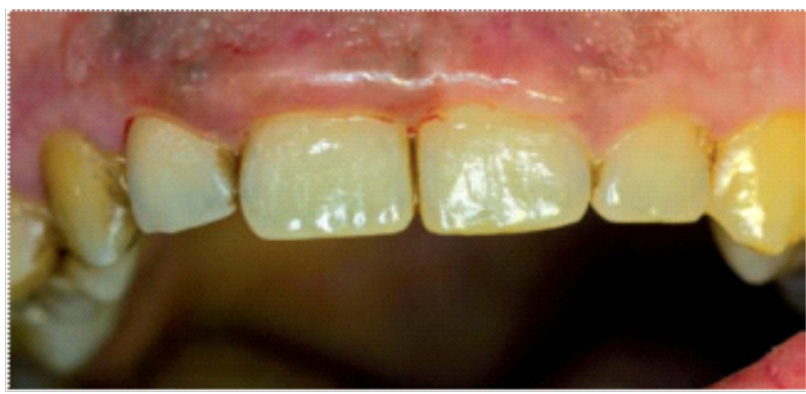

Figura 5. Laceración gingival

Fuente: Balda y cols. [5]

\section{Signo coronal}

\section{Proporción coronal alterada ancho/largo}

La literatura ha reportado para los incisivos centrales maxilares una proporción coronal ideal ancho/largo de $80 \%$, pero puede variar entre un $66 \%$ y un $80 \%$ (figura 6). Una proporción mayor se verá reflejada en un diente cuadrado, y una proporción menor en un diente largo [24-27]. 


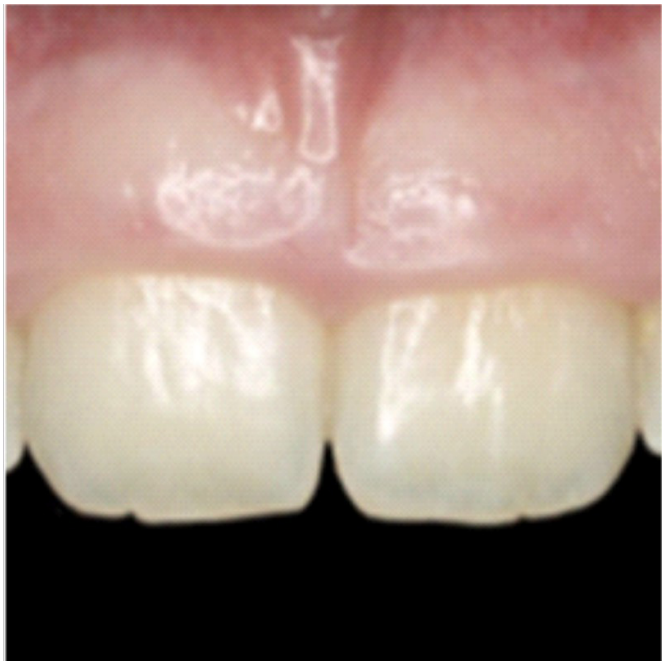

Figura 6. Adecuada proporción coronal ancho/largo Fuente: elaboración propia

En la erupción pasiva alterada, por estar el diente cubierto parcialmente por tejido gingival, se observa una reducción marcada de la longitud coronal encontrándose una proporción ancho/largo que está cerca de la unidad (proporción ancho/largo igual al 100\%), lo cual se refleja en un diente cuadrado (figura 7).

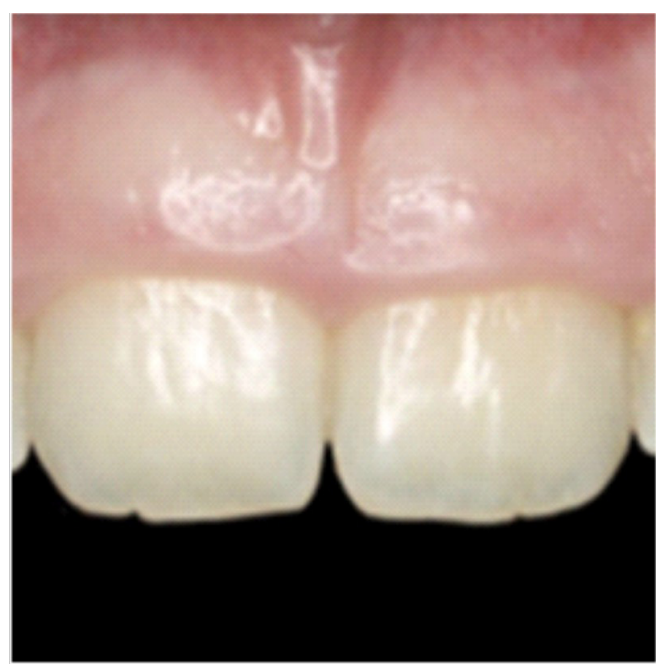

Figura 7. Proporción ancho/largo aumentada por erupción pasiva alterada (diente cuadrado)

Fuente: elaboración propia

\section{Edad del paciente}

La edad del paciente es un criterio clínico importante para diferenciar entre erupción pasiva alterada (EPA) y erupción pasiva retardada (EPR). La EPA se diagnostica cuando el paciente, por su edad, ya debería tener una adecuada exposición coronal, y no se debe esperar a que esta condición se corrija espontáneamente. La EPR se da cuando el paciente aún presenta una posición incisal del margen gingival pero, por su edad, todavía se puede esperar a que el margen gingival llegue a una posición adecuada sin necesidad de realizar procedimientos periodontales.

Para determinar la edad en que se puede hablar de erupción pasiva alterada se han realizado estudios como el de Volchansky y Cleaton-Jones [28], quienes evaluaron en modelos de estudio la posición del margen gingival de 237 niños caucásicos entre 6 y 16 años, encontrando que la posición del margen gingival cambió hasta los 12 años en los incisivos centrales inferiores, caninos, segundos premolares y primeros molares, mientras que para el resto de dientes hubo migración apical del margen gingival hasta los 16 años.

Sin embargo Morrow [29], en un estudio longitudinal más reciente, al hacer énfasis en el papel que juega la longitud de la corona en el aspecto estético, reporta que la longitud de la corona clínica de incisivos maxilares y caninos cambia 0,5 mm entre los 14-15 años y los 18-19 años. También indica que al parecer en la población femenina el proceso de erupción pasiva finaliza a los 18-19 años, mientras que en la población masculina parece que la erupción pasiva no termina a la misma edad. Aunque la información sirve como guía, con los resultados de este estudio no es posible determinar si los márgenes gingivales se estabilizan a la edad de 18-19 años.

Todo lo anterior sugiere que si después de los 19 años en promedio los dientes anteriores mantienen una proporción desfavorable entre la corona clínica y la anatómica, no se deben esperar mayores cambios, por lo que el clínico se puede encontrar frente a un proceso de erupción pasiva alterada y debe considerar el tratamiento de esta condición por medio de diferentes procedimientos plásticos periodontales [16].

\section{Diagnóstico y clasificación de la erupción pasiva alterada}

El diagnóstico de la erupción pasiva alterada se debe hacer buscando la diferencia de espacio que hay entre la unión cemento amélica y el fondo del surco, que en condiciones normales se puede hacer con un sondeo convencional, pues espera encontrarse al mismo nivel, pero en otros casos es necesario hacer sondeo con anestesia para localizar la unión cemento-amélica, 
ya que se puede encontrar apical al epitelio de unión. En esta situación, se realiza un sondaje hasta la cresta ósea para medir la distancia de la cresta ósea al LAC $\mathrm{y}$ al margen gingival. Cabe anotar que para tener un diagnóstico más claro, es necesario contar con radiografías periapicales, las cuales deben ser tomadas con técnica paralela, de esta manera se aprecia la longitud radicular, se localiza la cresta ósea a nivel interproximal y además se corrobora la localización del LAC [30].

Dependiendo de los resultados obtenidos del sondeo, se hace la clasificación de la erupción pasiva alterada de acuerdo con Coslet et al. [31], quienes en 1977 clasificaron la EPA en dos tipos teniendo en cuenta la cantidad de encía queratinizada, subdividiéndola en dos categorías según la relación entre la cresta ósea y el LAC (figura 8).

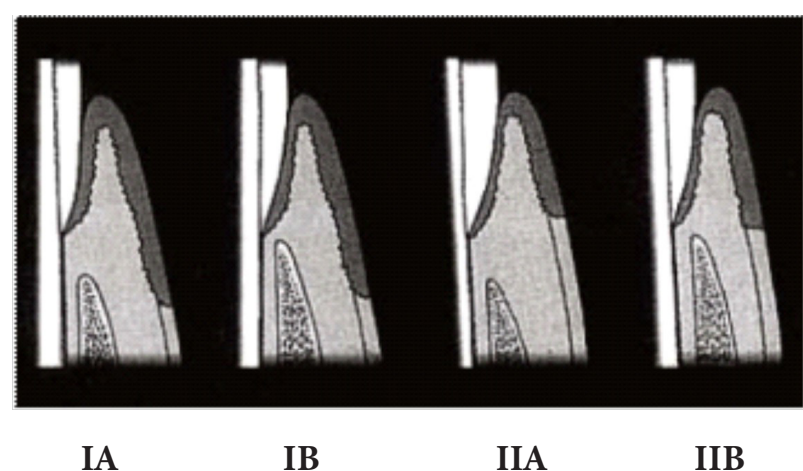

Figura 8. Clasificación de izquierda a derecha de la erupción pasiva alterada según Coslet et al.: tipo IA, tipo IB, tipo IIA y tipo IIB Fuente: Dolt [10]

- Tipo I: el margen gingival se ubica incisal u oclusal al LAC, y existe una amplia banda de encía desde el margen gingival hasta el límite mucogingival.

- Tipo II: la dimensión de la encía queratinizada tiene una amplitud normal localizándose sobre la corona anatómica, mientras que el límite mucogingival se localiza a nivel de la unión cemento-amélica.

- Subgrupo A: la distancia de la cresta ósea alveolar a la UCA es de $1,5 \mathrm{~mm}$ a $2,0 \mathrm{~mm}$.

- Subgrupo B: la cresta ósea se encuentra a nivel de la UCA.

\section{Manejo de la erupción pasiva alterada}

Los diferentes tipos de EPA se corrigen quirúrgicamente de manera diferente, como lo muestran Levine y McGuire [32] en su artículo de revisión en el que se describen de un modo detallado las técnicas quirúrgicas periodontales para el tratamiento de esta entidad, así:
- Tipo IA: gingivectomía a bisel externo.

- Tipo IB: gingivectomía con remodelado óseo.

- Tipo IIA: colgajo a bisel interno reposicionado apicalmente sin remodelado óseo.

- Tipo IIB: colgajo a bisel interno con remodelado óseo.

\section{Protocolo de tratamiento de la erupción pasiva alterada en el paciente ortodóncico}

Aunque generalmente se ha aceptado que el manejo de la erupción pasiva alterada se debe postergar hasta después de terminar el tratamiento ortodóncico, existen situaciones clínicas que muestran la necesidad de contar con un protocolo que determine si esta condición se debe manejar antes, durante o después de la ortodoncia, y especificar los tipos de procedimientos que se pueden hacer según la situación clínica y la edad del paciente.

\section{Antes}

En ortodoncia, por lo general no se plantean procedimientos plásticos periodontales antes de la ubicación de los brackets, aunque, en ocasiones, el inicio del tratamiento puede verse retrasado por una condición gingival $[10,33]$, como un proceso de erupción pasiva alterada, o por un proceso inflamatorio no controlado [34], que dificulta la colocación de estos.

Por tanto, la EPA se maneja antes de la ortodoncia cuando se encuentra un cubrimiento gingival coronal que impida lo colocación de dispositivos ortodóncicos.

En los pacientes adultos, independientemente del tipo de alteración en que se diagnostique (IA-IB-IIAIIB) su manejo, antes de la ortodoncia debe limitarse a eliminación o reposición de tejido blando, no se indica remoción ósea ya que la malposición dental inicial impide que se pueda realizar una correcta osteotomía.

Por otra parte, en pacientes adolescentes (menores de 19 años) todavía no se puede hablar de erupción pasiva alterada y, por tanto, además de la razón expuesta anteriormente en lo relacionado con la maloclusión, la edad es otro factor que hace que todos los procedimientos que se realicen se deban limitar a tejido blando, así este no sea un procedimiento estable.

Es importante aclarar que el hecho de limitar las intervenciones a tejido blando hace que aquellos casos que tengan o evolucionen (en caso de pacientes jóvenes) a una erupción pasiva alterada subtipo B (IB-IIB) deben ser re-intervenidos posteriormente, ya que no haber 
realizado la remoción ósea hace que durante el proceso de cicatrización la encía crezca de nuevo en sentido coronal para recuperar el espacio biológico [10, 29, 35].

\section{Durante}

Durante el tratamiento, el manejo de la erupción pasiva alterada en el paciente ortodóncico se debe realizar, en lo posible, específicamente cerca del final de la ortodoncia, cuando el clínico haya logrado la mayor cantidad de objetivos propuestos, y que el tiempo que va a durar con los aditamentos ortodóncicos sea el mínimo para poder evitar así un sobre crecimiento gingival causado por la irritación de los cementos y los brackets.

El objetivo fundamental del tratamiento de la EPA es que después del proceso de cicatrización el margen gingival cubra aproximadamente $1 \mathrm{~mm}$ de la corona anatómica del diente [10], resultado que favorece al ortodoncista para que pueda evaluar de manera directa y no radiográfica los márgenes y la posición de los cenit gingivales y determinar los ajustes que es necesario realizar en posición dental con el fin de lograr una adecuada estética del segmento anterior [36, 37].

\section{Manejo durante la ortodoncia de acuerdo con la edad del paciente}

Menores de 19 años: como se ha mencionado, en estos pacientes aún no se puede hablar de erupción pasiva alterada, pero en muchas ocasiones el clínico se enfrenta a coronas con gran cubrimiento gingival que le impiden finalizar adecuadamente los casos por la imposibilidad de visualizar directamente los cenit gingivales. Estos pacientes deben ser remitidos al periodoncista para que les realice sólo intervención en tejido blando, nunca de tejido duro, ya que por su edad aún se puede esperar una migración apical del hueso.

Es importante que se le aclare al paciente que es probable que este procedimiento no sea estable, por haberse limitado a tejido blando, y que puede ser necesario hacer otra intervención después de los 19 años, la cual dependerá del diagnóstico y la clasificación definitiva de la condición gingival. Mayores de 19 años: en estos pacientes el manejo dependerá del diagnóstico y la clasificación de la erupción pasiva alterada que presente, por tanto, se realizará manejo de tejido blando en los pacientes con EPA tipo IA y IIA, y manejo de tejido blando más remodelado óseo en los pacientes tipo IB y IIB. Es importante que se le aclare al paciente que aunque se le está realizando el procedimiento indicado para su condición, después de terminada la ortodoncia puede ser necesario tener que programar un retoque quirúrgico, generalmente de tejido blando, por las alteraciones que se puedan generar en la cicatrización debidas a los procesos inflamatorios relacionados con el tratamiento ortodóncico [38].

\section{Después}

Generalmente los pacientes que terminan el tratamiento ortodóncico presentan cierto grado de inflamación gingival que se refleja en un cubrimiento gingival coronal aumentado, que hace que el clínico deba esperar a que esta inflamación se resuelva para así poder determinar qué parte de ese cubrimiento coronal se debe al proceso inflamatorio, y qué parte a una falta de resolución completa de la EPA.

Generalmente los pacientes que terminaron su tratamiento de ortodoncia antes de los 19 años, y que mostraban un cubrimiento coronal gingival excesivo durante la ortodoncia - y en quienes fue necesario realizar un manejo de tejido blando para poder tener acceso a una adecuada longitud coronal - van a requerir después de los 19 años otro procedimiento plástico periodontal para corregir definitivamente su condición gingival, ya que algunos estudios - como el de Morrow [29] - han mostrado que entre los 14 y 19 años no hay gran migración apical en aquellos pacientes que presentaban una condición de erupción inadecuada. Por otra parte, en los pacientes adultos cuya EPA fue manejada durante la ortodoncia, es necesario evaluar la evolución de la posición gingival después de retirar los brackets, dando un tiempo de espera a que se resuelvan los cambios inflamatorios e hiperplásicos relacionados con la ortodoncia, los cuales son reversibles después de culminar el tratamiento, siempre y cuando sea implementado un adecuado protocolo de higiene oral [39]. Si después de que haya disminuido la inflamación se observa una posición gingival inadecuada, se debe remitir al periodoncista para que este realice procedimientos quirúrgicos que busquen mejorar los resultados obtenidos con la cirugía que se realizó durante la ortodoncia.

\section{Conclusiones}

- El ortodoncista debe estar en capacidad de diagnosticar y clasificar la erupción pasiva alterada, ya que esta condición puede comprometer los objetivos estéticos del tratamiento. 
- La edad es un factor determinante a la hora de realizar el diagnóstico y de ejecutar los procedimientos para el manejo de la erupción pasiva alterada.

- En personas menores de 19 años que presentan erupción pasiva retardada, se puede sospechar que evolucionen a una erupción pasiva alterada, y por tanto su abordaje se debe limitar sólo al manejo de tejido blando.

- El manejo de la erupción pasiva alterada en personas mayores de 19 años debe involucrar procedimientos de tejido blando con o sin remodelado del tejido óseo.

\section{Referencias}

[1] Gottlieb B, Orban B. Active and passive eruption of teeth. J Dent Res. 1933; 13: 214.

[2] Carranza F, Klokkevold P, Takei H, Newman M. Periodontología clínica. 10a. ed. México DF: McGraw-Hill; 2010.

[3] Gargiulo A, Wentz F, Orban B. Dimensions and relations of the dentogingival junction in humans. J Periodontol. 1961; 32(3): 261-7.

[4] Ainamo J, Loe H. Anatomical characteristics of gingiva. A clinical and microscopic study of the free and attached gingiva. J Periodontol. 1966; 37(1): 5-13.

[5] Balda y cols. www.actaodontologica.com/ediciones/1999/3/dolor_bucofacial_persistente.asp

[6] García I, Herrera J, Frías M, Carasol M. Erupción pasiva alterada. Implicaciones estéticas y alternativas terapéuticas. RCOE. 2006; 11(5-6): 563-71.

[7] Waldrop T. Gummy Smiles: The Challenge of Gingival Excess: Prevalence and Guidelines for Clinical Management. Seminars in Orthodontics. 2008; 14(4): 260-71.

[8] Davis NC. Smile design. Dent Clin North Am. 2007; 51(2): 299-318, vii.

[9] Dolt AH, 3rd, Robbins JW. Altered passive eruption: an etiology of short clinical crowns. Quintessence Int. 1997; 28(6): 363-72.

[10] Fernandez R, Arias J, Simonneau G. Erupción pasiva alterada. Repercusiones en la estética dentofacial. RCOE. 2005; 10(3): 289-302.

[11] Garber DA, Salama MA. The aesthetic smile: diagnosis and treatment. Periodontol 2000. 1996; 11: 18-28.

[12] Chu SJ, Karabin S, Mistry S. Short tooth syndrome: diagnosis, etiology, and treatment management. J Calif Dent Assoc. 2004; 32(2): 143-52.

[13] Henriques P. Estética en periodoncia y cirugía plástica periodontal. Brasil: Amolca; 2006.
[14] Armitage GC. Development of a classification system for periodontal diseases and conditions. Ann Periodontol. 1999; 4(1): 1-6.

[15] Foley TF, Sandhu HS, Athanasopoulos C. Esthetic periodontal considerations in orthodontic treatment--the management of excessive gingival display. J Can Dent Assoc. 2003; 69(6): 368-72.

[16] Krishnan V, Ambili R, Davidovitch Z, Murphy N. Gingiva and Orthodontic Treatment. Semin Orthod. 2007; 13(4): 257-71.

[17] Kokich VG. Esthetics and vertical tooth position: orthodontic possibilities. Compend Contin Educ Dent. 1997; 18(12): 1225-31; quiz 32.

[18] Kokich VG. Esthetics: the orthodontic-periodontic restorative connection. Semin Orthod. 1996; 2(1): 21-30.

[19] Kokich VG. Excellence in Finishing: Modifications for the Perio-Restorative Patient. Semin Orthod. 2003; 9(3).

[20] Fradeani M, Barducci G. Tratamiento protésico. Chigago: Quintessence, 2011.

[21] Casos clínicos/periodoncia. www.clinicavijande.com

[22] Townsend CL. Resective surgery: an esthetic application. Quintessence Int. 1993; 24(8): 535-42.

[23] Konikoff BM, Johnson DC, Schenkein HA, Kwatra N, Waldrop TC. Clinical crown length of the maxillary anterior teeth preorthodontics and postorthodontics. J Periodontol. 2007; 78(4): 645-53.

[24] Sarver DM. Principles of cosmetic dentistry in orthodontics: Part 1. Shape and proportionality of anterior teeth. Am J Orthod Dentofacial Orthop. 2004; 126(6): 749-53.

[25] Gillen R, Schwarts R, Hilton T, Evns D. An analysis of selective tooth proportions. Int J Prosthodont. 1994; 7: 410-7.

[26] Chu SJ. A biometric approach to predictable treatment of clinical crown discrepancies. Pract Proced Aesthet Dent. 2007; 19(7): 401-9; quiz 10.

[27] Chu SJ. Range and mean distribution frequency of individual tooth width of the maxillary anterior dentition. Pract Proced Aesthet Dent. 2007; 19(4): 209-15.

[28] Volchansky A, Cleaton-Jones P. The position of the gingival margin as expressed by clinical crown height in children aged 6-16 years. J Dent. 1976; 4(3): 116-22.

[29] Morrow LA, Robbins JW, Jones DL, Wilson NH. Clinical crown length changes from age 12-19 years: a longitudinal study. J Dent. 2000; 28(7): 469-73.

[30] Hempton TJ, Dominici JT. Contemporary crownlengthening therapy: a review. J Am Dent Assoc. 2010; 141(6): 647-55. 
[31] Coslet JG, Vanarsdall R, Weisgold A. Diagnosis and classification of delayed passive eruption of the dentogingival junction in the adult. Alpha Omegan. 1977; 70(3): 24-8.

[32] Levine RA, McGuire M. The diagnosis and treatment of the gummy smile. Compend Contin Educ Dent. 1997; 18(8): 757-62, 64; quiz 66.

[33] Sarver DM, Yanosky M. Principles of cosmetic dentistry in orthodontics: Part 3. Laser treatments for tooth eruption and soft tissue problems. Am J Orthod Dentofacial Orthop. 2005; 127(2): 262-4.

[34] Mariotti A. Dental plaque-induced gingival diseases. Ann Periodontol. 1999; 4(1): 7-19.

[35] Jorgensen M, Nowzari H. Aesthetic crown lengthening Periodontol 2000. 2001; 27: 45-58.
[36] Lee J. The effects of buccolingual root torque on the appearance of root angulation on panoramic radiographs. Am J Orthod Dentofacial Orthop. 2005; 27(3): 393.

[37] Owens AM, Johal A. Near-end of treatment panoramic radiograph in the assessment of mesiodistal root angulation. Angle Orthod. 2008; 78(3): 475-81.

[38] Naranjo AA, Trivino ML, Jaramillo A, Betancourth M, Botero JE. Changes in the subgingival microbiota and periodontal parameters before and 3 months after bracket placement. Am J Orthod Dentofacial Orthop. 2006; 130(3): 275 e17-22.

[39] Kloehn JS, Pfeifer JS. The effect of orthodontic treatment on the periodontium. Angle Orthod. 1974; 44(2): 127-34. 